\title{
Probing the Geometry and Physics of the Emission Region of Active Galactic Nuclei Using Hard X-ray Observations
}

\author{
Natasha Woods \\ Office of Science, Science Undergraduate Laboratory \\ Internship (SULI) \\ The University of Texas at Dallas \\ Stanford Linear Accelerator Center \\ Menlo Park, CA \\ August 13, 2009 \\ Prepared in partial fulfillment of the requirements of the Office \\ of Science, Department of Energy's Science Undergraduate \\ Laboratory Internship under the direction of Marco Ajello and \\ Masaaki Hayashida at the Kavli Institute for Particle \\ Astrophysics and Cosmology, Stanford Linear Accelerator \\ Center.
}

Participant:

Signature

Research Advisors:

Signature

Signature 


\section{Contents}

1 Introduction $\quad 4$

1.1 AGN Classification . . . . . . . . . . . . . . . . 4

1.2 Unified Model for AGN . . . . . . . . . . . . . . . . . 4

1.3 Investigating the Unified Model of AGN using X-rays . . . . . 5

1.4 Relevance ................... . . . 6

$\begin{array}{llr}2 & \text { Methods } & 7\end{array}$

2.1 Swift-BAT Mission . . . . . . . . . . . . . . . . 7

2.2 Sample Population . . . . . . . . . . . . . . . . 7

2.3 Data Analysis . . . . . . . . . . . . . . . . . . 7

2.3.1 Photon Indices \& Simple Power Law Model . . . . . . 7

2.3.2 Stacked Spectra . . . . . . . . . . . . 8

$\begin{array}{llr}3 & \text { Results } & 9\end{array}$

4 Discussion \& Conclusion $\quad 10$

5 Acknowledgments $\quad 11$

$\begin{array}{llr}6 & \text { Figures } & 14\end{array}$ 


\begin{abstract}
Probing the Geometry and Physics of the Emission Region of Active Galactic Nuclei Using Hard X-ray Observations. NATASHA L. WOODS (University of Texas at Dallas, Richardson, TX 75080) MARCO AJELLO \& MASAAKI HAYASHIDA (Kavli Institute for Particle Astrophysics and Cosmology, Stanford Linear Accelerator Center, Menlo Park, CA 94204)
\end{abstract}

The X-ray spectra of $\sim 200$ AGN collected from Swift-BAT were analyzed to test the Unified Model for AGN. Specifically, the photon indices, high energy cutoffs, and reflection components of Sy1 and Sy2 were compared. Under the Unified Model, the photon indices and reflection components for Sy1 should be larger than Sy2 and the high energy cutoffs should be the same. Fitting a simple power law model to the sample spectra proved to be insufficient. The PEXRAV model fit the spectra of the Sy1 and Sy2 significantly better, indicating that a reflection component and/or high energy cutoff exists as the Unified Model expects. Using both the simple power law and PEXRAV models it was concluded that in the population studied, Sy1 had a larger photon index than Sy2, as expected by the Unified Model. For Sy1 and Sy2, the reflection components were found to be compatible, but given the large errors, this finding cannot be said to be evidence against the Unified Model. However, it was concluded that Sy1 and Sy2 have different high energy cutoffs, which is unexpected under the Unified Model. 


\section{Introduction}

The study of Active Galactic Nuclei (AGN) began in 1908 when Arthur Fath noticed six emission lines in the spectra of NGC 1068 that could not be attributed to stellar processes. Less than two decades thereafter, Carl Seyfert recognized similar emission lines in the spectra of spiral galaxies (Fig.1). Over time, galaxies with these emission lines were named AGN. These objects are characterized by the fact that the majority of their energy output is unrelated to stellar processes. Formally, AGN are compact regions of space at the center of galaxies that are exceedingly luminous over all or part of the electromagnetic spectrum [1].

\subsection{AGN Classification}

AGN are classified by their radio luminosity and spectral properties. Radioloud AGN emit radio waves, radio-quiet AGN have minimal or no radio flux. Additionally, if the source has a bright optical continuum with broad emission lines, it is classified as Type 1. Type 2 AGN have a weak optical continuum with narrow emission lines [2].

\subsection{Unified Model for AGN}

The Unified Model for AGN posits that all AGN are intrinsically the same and their perceived differences can be attributed to their viewing orientations. In this model, AGN are axis symmetric and host $10^{6-10} M_{\text {sun }}$ super-massive black holes (SMBH) that actively accrete matter from surrounding accretion disks. Outside this disk is a molecular torus composed of gas and dust (Fig.2). The viewing orientation of the torus is believed to obscure or reveal the luminous continuum emission from the SMBH and its accretion disk.

AGN have a broad line region (BLR) composed of 3,000-30,000K gas clouds orbiting the SMBH at 3,000-10,000 km/s, 0.05pc from the SMBH. Doppler shifting of the photons emitted from the BLR broadens emission lines from this region. The narrow line region (NLR) consists of gas clouds also orbiting the SMBH, but 100-300pc from the black hole at 100-300 km/s, producing much narrower emission lines (Fig. 2) [1]. In this work, Seyfert galaxies, a class of radio-quiet AGN were studied. In Seyfert 1 galaxies (Sy1) the torus is viewed face-on making both the NLR and BLR visible. Conversely, in Seyfert 2 galaxies (Sy2) the torus is viewed edge-on and only 
the NLR is usually observed (Fig.3) [1]. Much evidence has been found to support this model. Antonucci and Miller found polarized broad emission lines in Sy2 spectra in 1985, evidence that Sy2 had a BLR hidden by the torus [13]. Additionally, the luminosity, rapid energy variability, and velocity dispersion of stars near SMBHs best match the observations of the central engine of AGN [1].

However, there are observations which are inconsistent or unexplained in this model. For instance, AGN have been observed to change from Sy1 to Sy2 and vice versa [3]. According to the Unified Model, the classification of AGN depends only on orientation, which is constant. Therefore, AGN cannot change classes under this model. This suggests that other factors also determine AGN classification, such as the density and motion of material in the torus.

Another piece of contradictory evidence is the possible difference in electron temperatures in the coronas of Sy1 and Sy2 [1]. If the electron temperatures are truly different, the magnetic fields of Sy1 and Sy2 might be different, which the Unified Model does not mention. Essentially, this would suggest that these objects are not the same, namely their differences could not be attributed to their orientation alone, as the Unified Model states.

Additionally, the fraction of obscured sources might depend on luminosity as well as orientation [3]. If this is true, more luminous objects would tend to be unobscured due to their increased luminosity, not their orientation alone.

\subsection{Investigating the Unified Model of AGN using X- rays}

To further investigate this model, the X-ray spectra of the sample population, which probes the innermost regions of AGN as the torus is transparent to XRays $^{1}$, was examined. X-ray emission from AGN is believed to be a product of inverse Compton radiation (IC). In this process, photons from the accretion disk are up-scattered by hot (possibly relativistic) electrons in a corona above the disk in the X-ray energy band [1]. The origin and exact geometry of the hot corona remains highly debatable and was further examined.

The IC light observed from AGN falls into three categories: direct, trans-

\footnotetext{
${ }^{1}$ The torus of a Compton-thick source, a source where $\mathrm{N}_{H} \sim 1.5 \times 10^{24} \mathrm{~cm}^{-2}\left(\mathrm{~N}_{H}\right.$ is the density of neutral Hydrogen atoms), is not transparent to X-Rays. However, only $5 \%$ of the sample population was Compton-thick.
} 
mitted, and reflected. Direct light comes from photons scattered off the corona. Transmitted light originates from photons scattered off the corona that travel through the torus. Reflected light is scattered off the corona and reflected off the accretion disk or torus via inverse Compton reflection. This type of reflection peaks at $30 \mathrm{keV}$ and then sharply declines. Consequently, the larger the reflection component the softer the spectrum in the 15-200 $\mathrm{keV}$ band. Due to the mere geometry of the model, Sy1 should have a larger reflection component than Sy2 in the Unified Model [7].

The reflection component of the total light emitted by AGN and the photon index (see Methods) are directly related. By examining the distribution of the photon indices, the Unified model was tested. Under the Unified Model the photon index of Sy1 should be larger than that of Sy2 as Sy1 galaxies have a larger reflection component.

A high energy cutoff is expected in both Sy1 and Sy2 spectra as the corona's electrons, which IC scatter the photons, are expected to be thermal with their own high energy cutoff, due to observations such as Fe emission lines. Moreover, the cutoff for Sy1 and Sy2 should be compatible since the properties of the corona should be independent of orientation.

\subsection{Relevance}

Ultimately, the Unified Model for AGN was tested. The photon indices, high energy cutoffs, and reflection components of Sy1 and Sy2 galaxies were compared to determine if Sy1 and Sy2 are intrinsically different. This research matters as AGN are believed to account for $100 \%$ of the Cosmic X-ray Background (CXB) (Fig.4). However, for this to be true, it is believed that a third of the AGN population should be Compton-thick, and very few of these objects have been detected thus far. Another explanation of the CXB posits that AGN have a larger reflection component than previously estimated, and the sum of all AGN reflection peaks at $\sim 30 \mathrm{keV}$ will reproduce the CXB without the need of more Compton-thick sources. Additionally, better understanding AGN and their relationship to the CXB will help place constraints on cosmological models and black hole physics. 


\section{Methods}

\subsection{Swift-BAT Mission}

Swift is a revolutionary multi-wavelength observatory focused on the GammaRay Burst science (GRB). Launched in 2004, Swift uses three instruments: the Burst Alert Telescope (BAT), the X-ray Telescope (XRT), and the Ultraviolet/Optical Telescope (UVOT), to monitor GRBs and afterglows in gamma-ray, X-ray, optical, and ultraviolet wavebands. BAT, the largest instrument on board, detects $\sim 100$ GRBs per year. Once a GRB is detected, XRT and UVOT are aimed at the burst to help determine the location and spectrum of the GRB. The data collected is circulated publicly worldwide for follow-up observations and studies. BAT is a coded mask telescope that utilizes a photon-counting CdZnTe detector with a $5200 \mathrm{~cm}^{2}$ detecting area in the $15-150 \mathrm{keV}$ range [14]. BAT surveys the X-ray sky with unprecedented sensitivity due to its wide field of view and pointing strategy enabling it to continuously monitor $\sim 80 \%$ of the sky daily. It has reached a sensitivity of $\sim 1 \mathrm{mCrab}$ in $1 \mathrm{Ms}$ of exposure, making it a perfect instrument for studying objects whose emission is faint in hard X-rays such as AGN [13]. The raw data from the telescope is calibrated using various calibration files such as the two seen in Fig. $5 \& 7$.

\subsection{Sample Population}

All the sources in this sample were detected above a $5 \sigma$ significance level from a three year exposure from the Swift-BAT hard X-ray survey (see [13] for more information). As mentioned before, the sources were studied in the 15-200 keV range, in which photons are transparent to the torus, further strengthening the integrity of this sample. This study was different from previous work in that an unbiased sample (blind search) of $\sim 200$ sources from [13] was used (See Fig.6\&8 and Table 6).

\subsection{Data Analysis}

\subsubsection{Photon Indices \& Simple Power Law Model}

Once the sources were chosen, spectra were generated by the methods specified in [13]. Using these spectra, photon indices for each source were determined by fitting the simple power law model to the sample spectra seen in 
Equation (1) using $\mathrm{X}_{\mathrm{spec}}$ 11.3.2. This program fits the data to Equation (1) using the $\chi^{2}$ statistic for the data by varying model parameters (see Fig.12).

$$
\frac{d N}{d E}=A E^{-\Gamma}
$$

In Equation (1) $\Gamma$ is the photon index, $\mathrm{A}$ is the normalization, $\mathrm{N}$ is the number of photons, and $\mathrm{E}$ is energy.

A python script was then written that used Root 5.18 to generate a photon index vs. redshift plot and a photon index histogram for the sample population (see Fig.9\&11). The Gaussian distribution was then fit to the generated histograms using the Binned Likelihood and $\chi^{2}$ methods in Root (see Table 3). Using the fitted histogram, the mean photon index, its error and sigma for Sy1, 1.5, 2 and other sources were determined.

Additionally, weighted averages and their errors were computed for Sy1 and Sy2. This method is particularly applicable in this case as all the individual errors are accounted for and this average does not involve fitting a model to the data.

To decide if the mean photon indices of Sy1 and Sy2 were statistically different, the Kolmogorov-Smirnov test, a non-parametric test, meaning that no assumptions are made about the data's probability distributions, was used. This test determines if two samples come from the same parent population by determining the maximum distance between the two samples' cumulative distribution functions. The p-value of this test was determined by using the same python script mentioned above.

To further ensure the validity of these results, the sample population was then divided into two groups around the mean flux, $2.89 \times 10^{-11} \mathrm{ergs} / \mathrm{cm}^{2} / \mathrm{s}$. The sources that had a flux greater than the mean were classified as bright. The sources with a flux less than or equal to the mean flux were classified as faint. The same analysis listed above for the entire population was then repeated for the faint and bright sources.

\subsubsection{Stacked Spectra}

To further investigate the Unified Model stacked spectra were created and examined. A stacked spectrum is a weighted average of individual spectra based on their errors. By using stacked spectra, the signal to noise ratio $(\mathrm{S} / \mathrm{N})$ increases and the mean properties of populations are more easily studied. Stacked Spectra for Sy1 and 2 were generated. The data for the stacked 
spectra was calculated using another python script that was created.

The simple power law and the PEXRAV models were fit to the Sy1 and Sy2 stacked spectra using Xspec. Utilizing the simple power law model the mean photon index, its error, and sigma were determined. The PEXRAV model, unlike the simple power law model, adds the reflection component and high energy cutoffs to the underlying continuum providing a better fit the majority of the time. First, the stacked spectra were fit ignoring the high energy cutoff, and the photon index and reflection component were determined. The reflection component is the total amount of reflection observed normalized to the directly viewed primary spectrum based on their associated integrated power outputs. When using this model Sy1 and Sy2 were assumed to be at inclination angles ${ }^{2}$ of 30 and 60 degrees, respectively. Then the reflection component was ignored, and the high energy cutoff and photon index were determined. For Sy2 the photon index, reflection component, and high energy cutoff were fit simultaneously as neither of the two previous fits accurately described the data.

\section{Results}

The results from fitting the histograms generated by the simple power law model from all the individual spectra and from the calculated weighted averages and errors are seen in Table 3. The p-values of the Kolmogorov-Smirnov test for Sy1 and Sy2 are listed in Table 2. The weighted averages were more accurate in this instance as significant tails in the histograms caused the Gaussian to not fit the data well (see Fig.10) and the individual errors for all the AGN in the sample are accounted for in this method.

The results of fitting the stacked spectra to the PEXRAV model and the simple power law model are seen in Table 4 . The $\chi^{2}$ values for the PEXRAV model were significantly smaller than those for the simple power law model for the stacked spectra in Table 4 .

The results from both Table $3 \& 4$ showed that the spectra of Sy1 are softer than that of Sy2, meaning the Sy1 have a larger photon index than Sy2. Sy2 seemed to need a high energy cutoff $\sim 360 \mathrm{keV}$, whereas Sy1 had a high energy

\footnotetext{
${ }^{2}$ The inclination angle is the angle between the normal of the accretion disc and the line of sight.
} 
cutoff outside the X-ray energy band. Finally, the reflection components of Sy1 and Sy2 were found to be compatible within their errors.

\section{Discussion \& Conclusion}

From the photon index histograms generated from the individual spectra, it is evident that the mean photon indices of Sy1 and Sy2 are different at a $2 \sigma$ level (Table 2), specifically Sy1 had a larger photon index than Sy2. This trend is in agreement with [3]. Under the Unified Model the mean photon index of Sy1 should be larger than that of Sy2 as the reflection component in Sy1 is larger than that in Sy2, which increases the photon index.

When examining the data from the stacked spectra, it is evident that the PEXRAV model fits the spectra significantly better than the simple power law model based on the lower $\chi^{2}$ values. This indicates that AGN probably do have a high energy cut and/or reflection component, in agreement with the Unified Model. However, accounting for these new parameters did not significantly change the photon index, as the photon index for Sy1 was still larger than that of Sy2 when this model was fit to the stacked spectra.

The reflection component for Sy1 under the Unified Model should be larger than that of Sy2. However, the data in Table 4 indicates that the reflection components for Sy1 and Sy2 are compatible, but given the significant errors, this finding does not disprove the Unified Model. Moreover, Sy2 seemed to have a high energy cutoff at $\sim 360 \mathrm{keV}$, whereas Sy1 had a cutoff

outside the X-ray energy band, suggesting the corona of Sy1 and Sy2 are different, which is not in agreement with the Unified Model.

Overall, in this work the photon index of Sy1 was found to be larger than that of Sy2, as the Unified Model predicts. However, Sy1 and Sy2 had compatible reflection components. Given the significant errors of this finding, this cannot be said to be evidence against the Unified Model. However, it was concluded that Sy1 and Sy2 do have different high energy cutoffs, which is not in agreement with the Unified Model. Given the substantial errors of parts of the data, to make more definite conclusions a larger sample should be used. This is plausible as the data for this study came from Swift-BAT, which is still accumulating data. 


\section{Acknowledgments}

This research, conducted at the Stanford Linear Accelerator Center, could not have been completed without the support, funding, and opportunity provided by the Department of Energy, Office of Science, SLAC, and KIPAC. My mentors, Marco Ajello and Masaaki Hayashida deserve much thanks for their patience, enthusiasm, and expertise. I would also like to thank all the SULI students and staff at SLAC. 


\section{References}

[1] M. Ajello, "Swift/BAT studies of AGN and the Cosmic X-ray Background", Universittsbibliothek der TU Mnchen, 2007.

[2] O. Labiano, "Host galaxies and environments of compact extragalactic radio sources", Kapteyn Astronomical Institute and Space Telescope Science Institute, February 2006.

[3] V. Beckmann, S. Soldi, C. Ricci, J. Alfonso-Garzn, T.J.-L. Courvoisier, A. Domingo, N. Gehrels, P. Lubinsk i, J. M. Mas-Hesse, and A. A. Zdziarski, "The Second INTEGRAL AGN Catalogue", July 2009

[4] S. Kraemer, R. Windhorst, K. Carpenter, M. Crenshaw, M. Elvis,and M. Karovska, "Active Galactic Nuclei and their role in Galaxy Formation and Evolution", February 2009.

[5] P.O. Petrucci, "Models of Comptonization", HAL - CCSD, 2008.

[6] V. Beckmann, S. Soldi, C.R. Shrader, N. Gehrels, and N. Produit, "The Hard X-ray 20-40 keV AGN Luminosity Function”, 2006.

[7] M. Ajello, A. Rau, J. Greiner, G. Kanbach, M. Salvato, A. W. Strong, S. D. Barthelmy, N. Gehrels, C. B. Mar kwardt, J. Tueller, "BAT X-ray Survey - III: X-ray Spectra and Statistical Properties", The Astrophysical Journal, September 2007.

[8] S. Deluit, T. Courvoisier, "The intrinsic emission of Seyfert galaxies observed with BeppoSAX/PDS I. Comparison of the average spectra of the three classes of Seyfert Galaxies", Astron. Astrophys. 399 77, 2003.

[9] P. Ricker and P. Meszaros, "Starburst and Reflection-Dominated AGN Contributions to the Cosmic X-Ray Background", ApJ vol. 418 49, 1993.

[10] C. Caradamone, http://www.astro.yale.edu/ccardamone/index.html

[11] D.E. Gruber, J. Matteson, L. Peterson, and G. Jung, "The Spectrum of Diffuse Cosmic Hard X-Rays Measured with HEAO-1", 1999.

[12] P. Magdziarz, A.A. Zdziarski, "The nature of the X-ray source in NGC 415”, 1995. 
[13] M. Ajello, L. Costamante, R. M. Sambruna, N. Gehrels, J. Chiang, A. Rau, A. Escala, J. Greiner, J. Tueller, J. V. Wall, and R. F. Mushotzky, "The Evolution of Swift/BAT blazars and the o rigin of the MeV background", ApJ, 2009.

[14] BAT Digest, swift.gsfc.nasa.gov, 2005.

[15] Zwillinger D.\& Kokoska S., Standard Probability and Statistics Tables and Formulae. Boca Raton, Florida: CRC Press LLC, -c2000, ISBN 1-58488-059-7. 


\section{$6 \quad$ Figures}

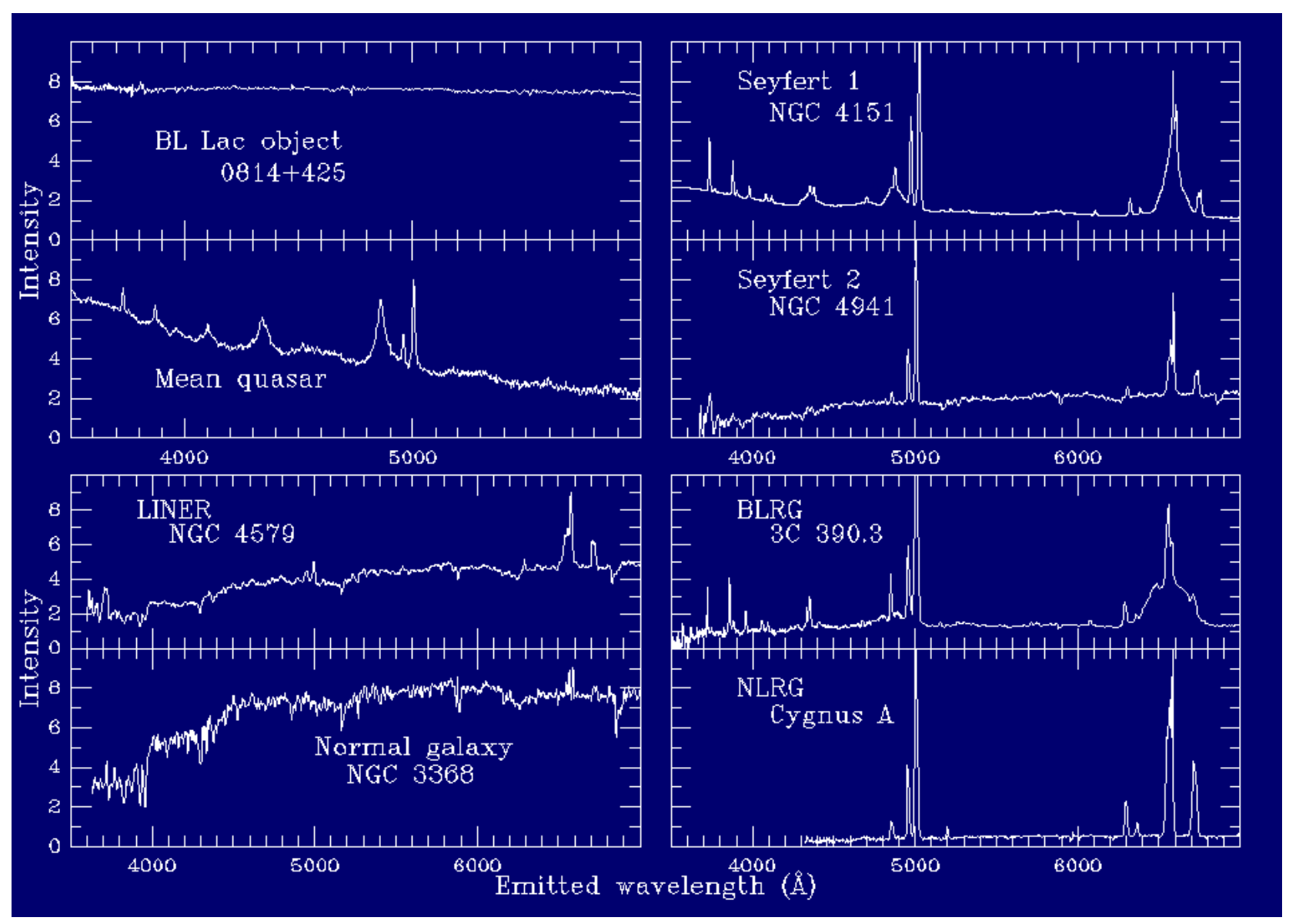

Figure 1: Representative spectra of different types of AGN. The Seyfert 1 spectrum has noticeably broader emission lines than Seyfert 2 spectrum. Image from Ajello [1]. 


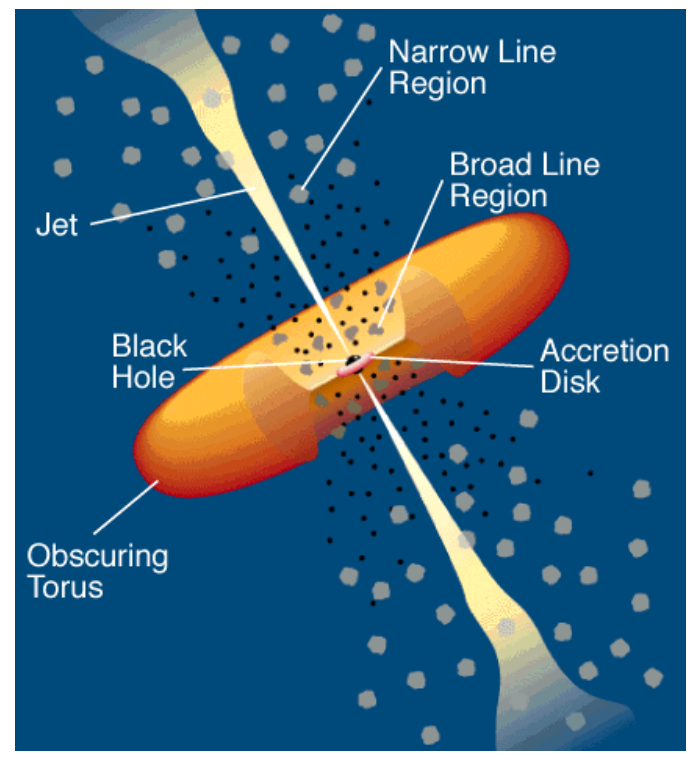

Figure 2: Unified Model. Image from Ajello [1].

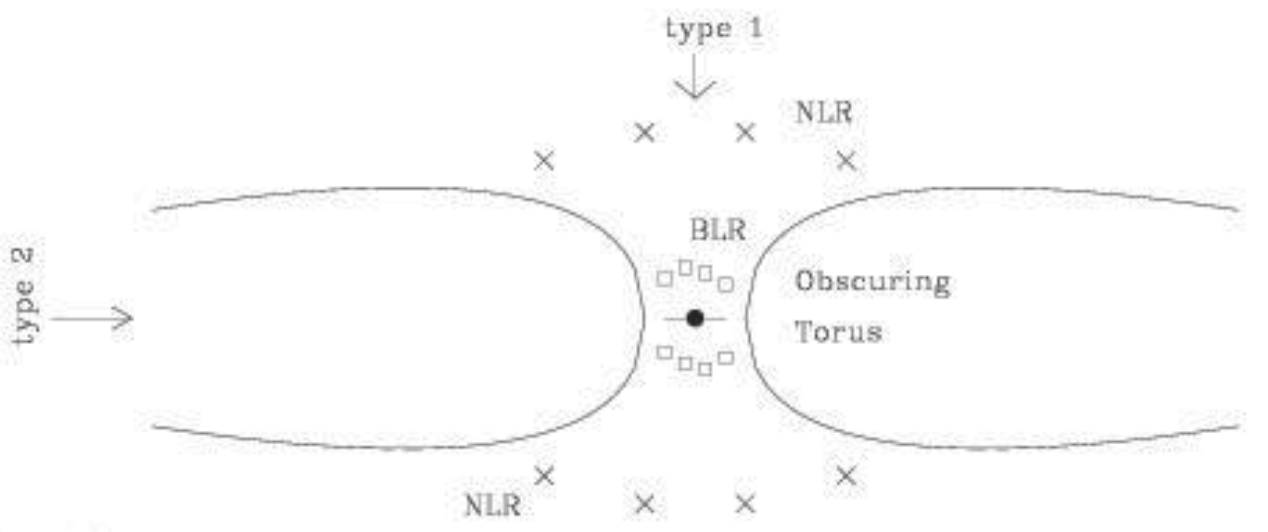

Figure 3: In Sy2 the torus is viewed more edge-on. In Sy1 the torus is viewed more face-on. Image from Cardamone [10]. 


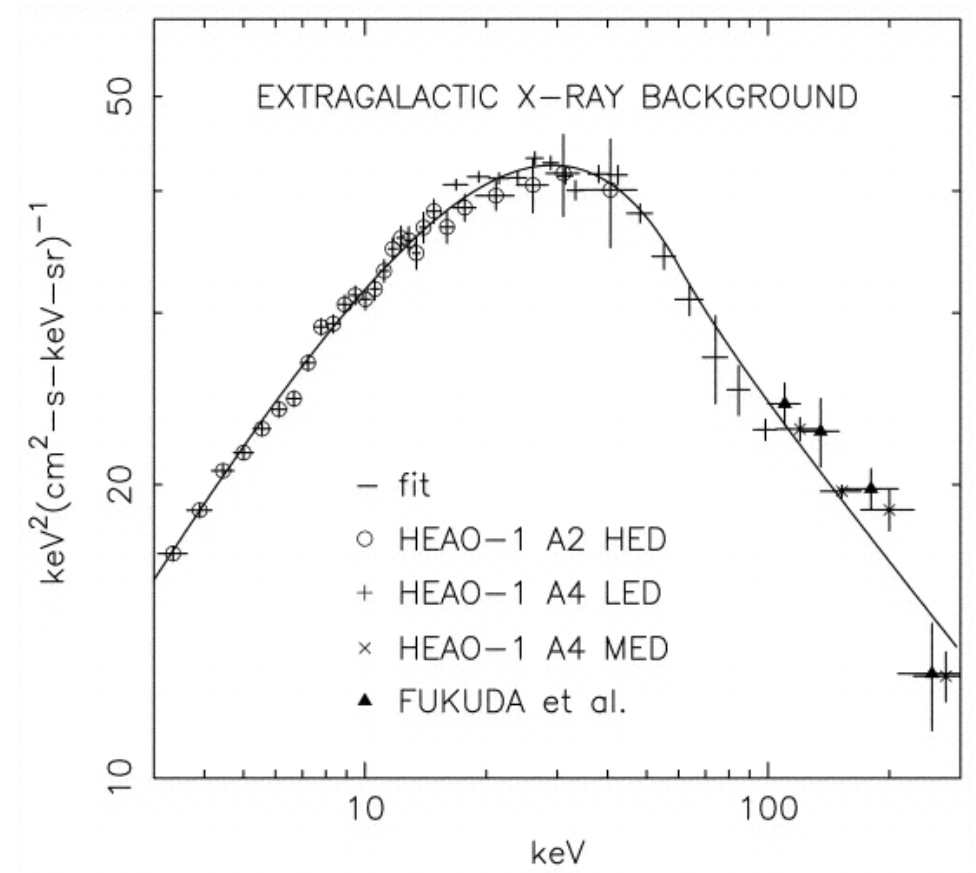

Figure 4: Photon spectrum of CXB compiled from various instruments on HEAO 1. Image from Gruber [11].

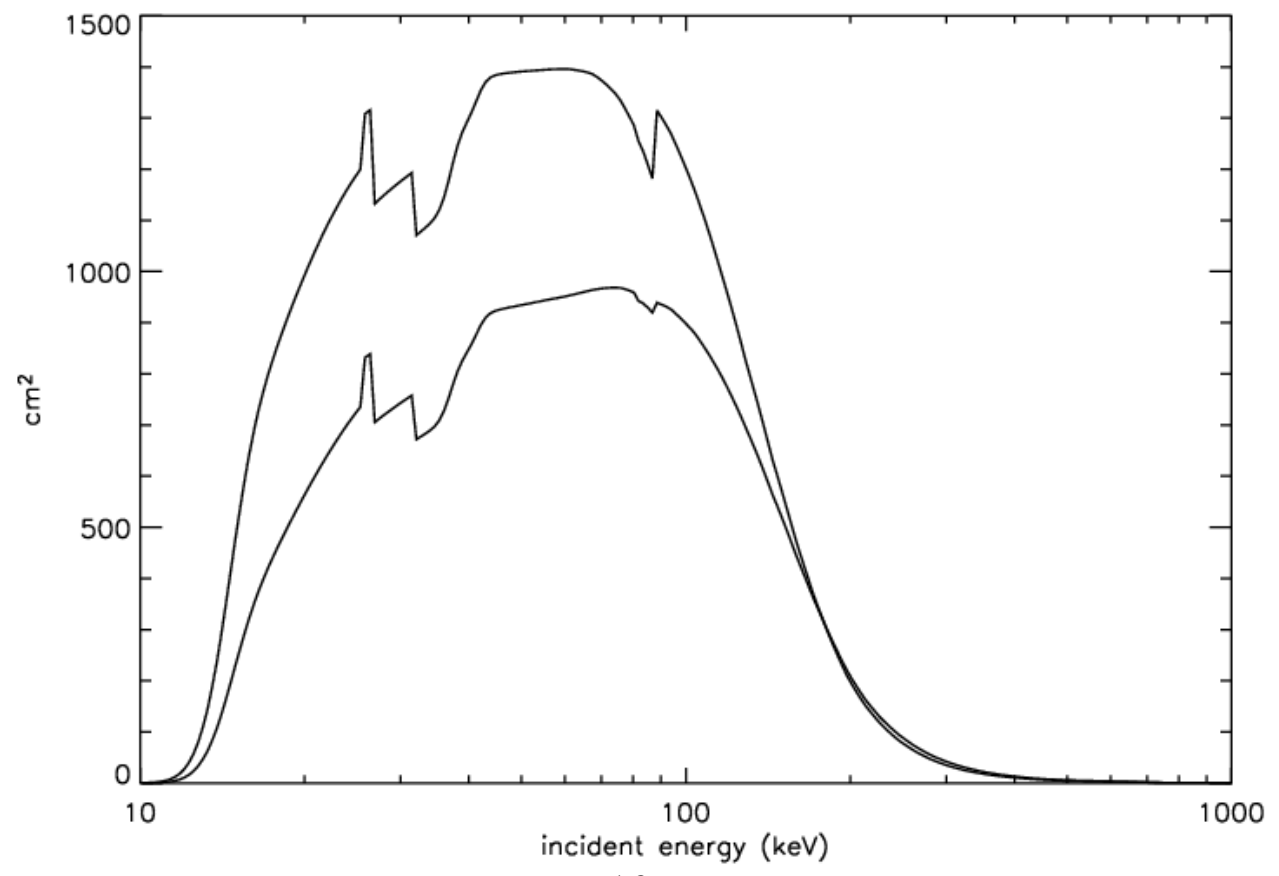

16

Figure 5: Effective area of the BAT for on- and off-axis (45 deg.) sources at different energy levels [14]. 


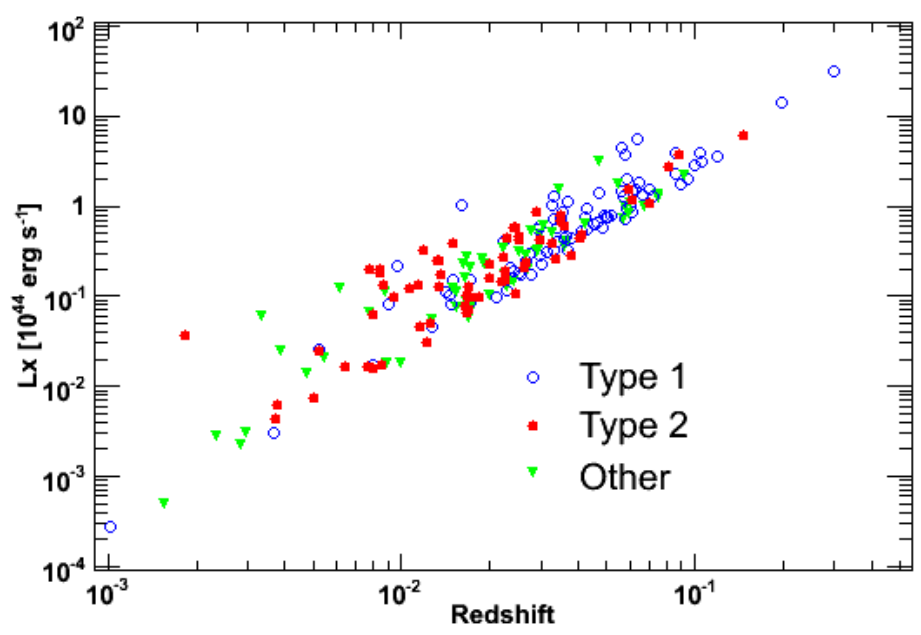

Figure 6: Luminosity vs. Redshift of source population studied.

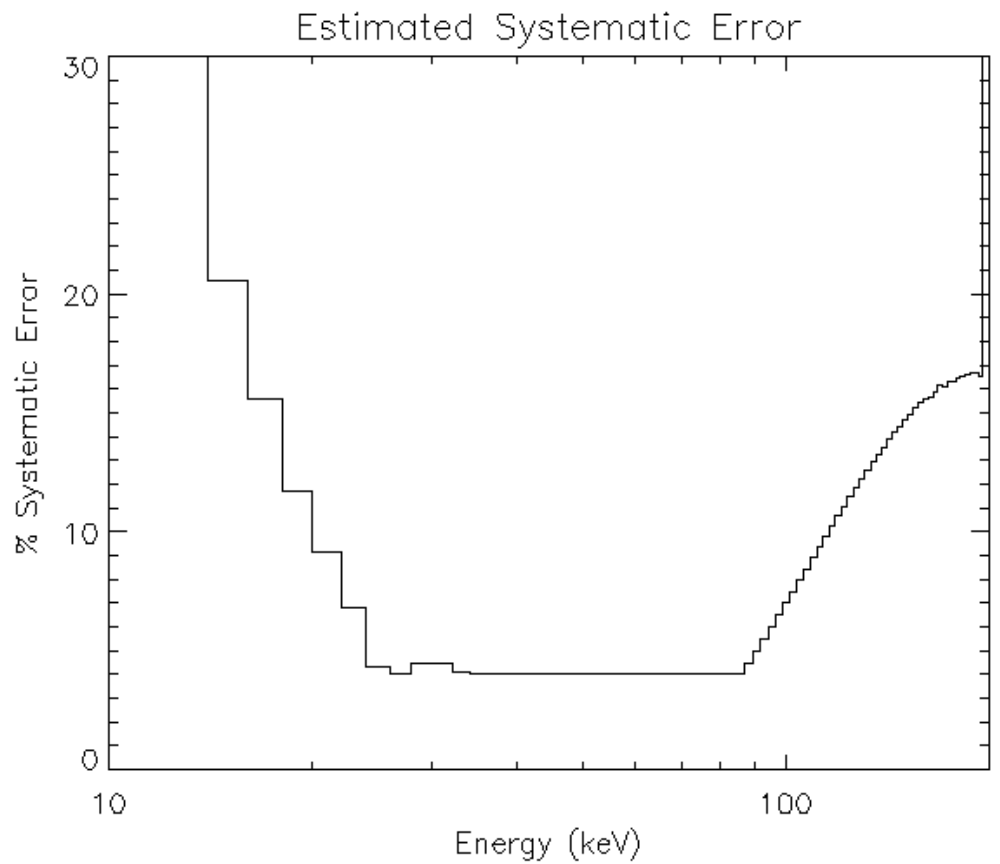

Figure 7: Systematic error of BAT as a function of energy [14]. 


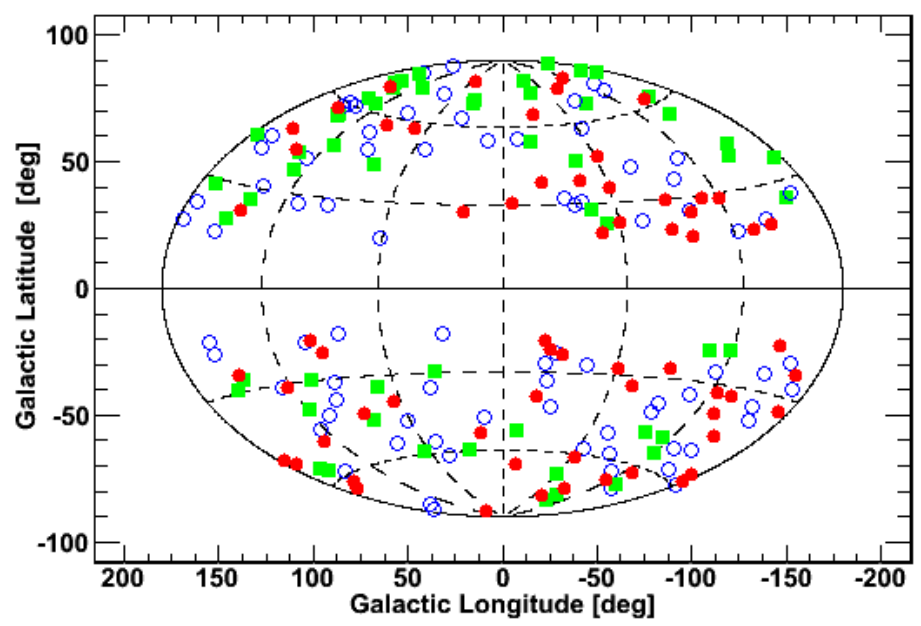

Figure 8: Map of source population.

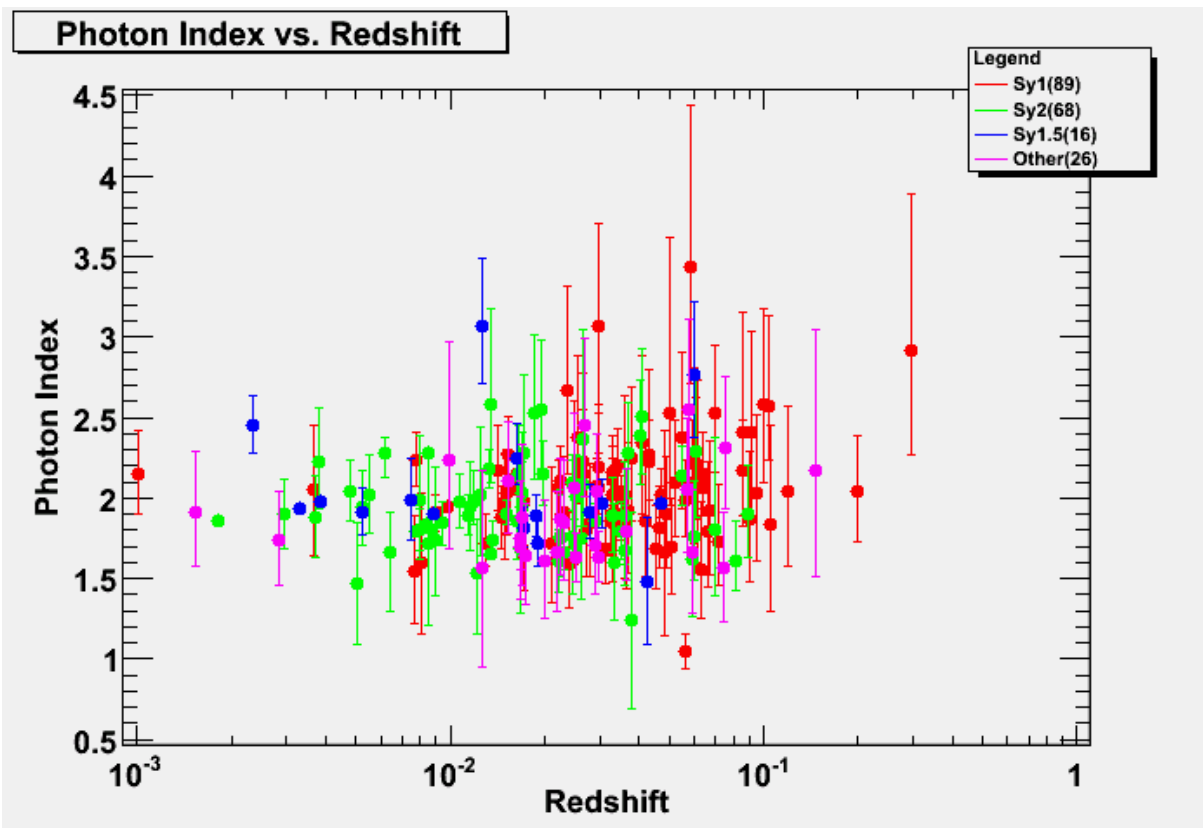

Figure 9: Redshift vs. Photon Index of source population generated using Root. 


\section{Number of Sources vs. Photon Index}

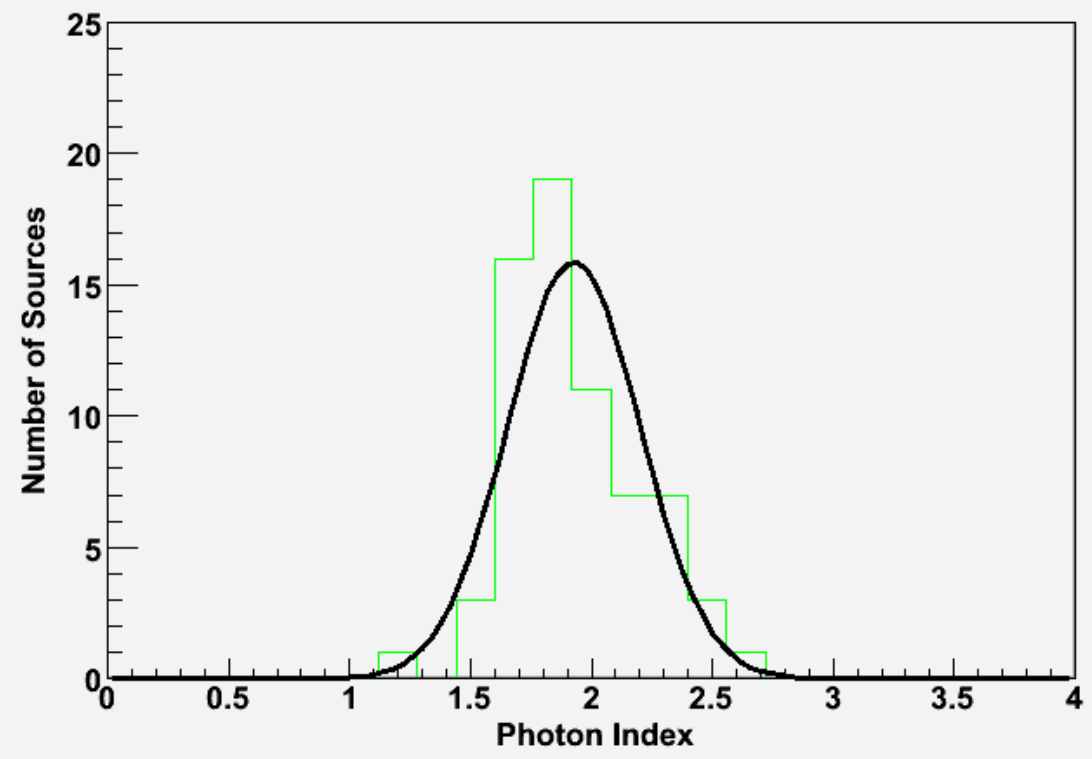

Figure 10: Gaussian Fit of entire Sy2 population using binned likelihood method. The right-hand tail of the data skews the data, causing the Gaussian to not match the data well.

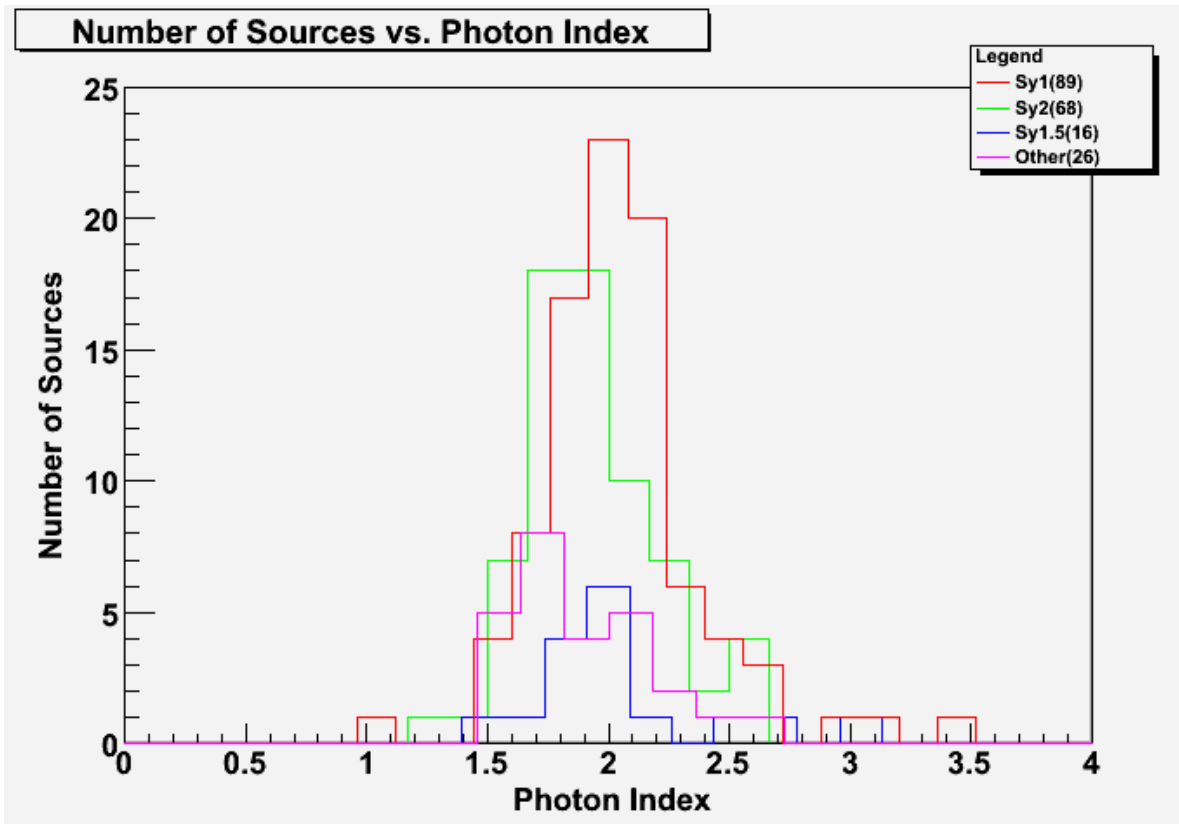

Figure 11: Sample population distribution generated using Root. 


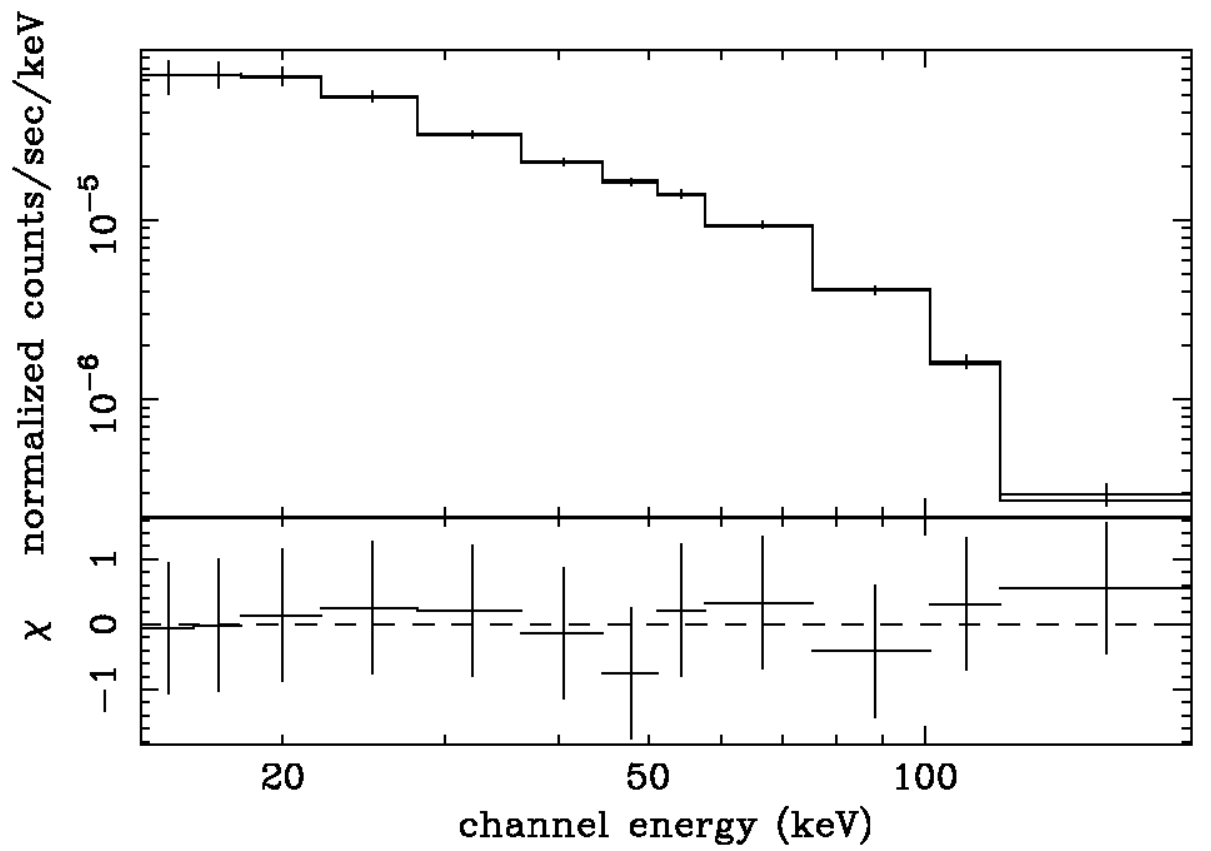

Figure 12: Typical spectra generated by Xspec11.

\begin{tabular}{ccccc}
\hline \hline Type & Sy1 & Sy1.5 & Sy2 & Others \\
\hline Number of Sources & 89 & 16 & 68 & 26 \\
\hline
\end{tabular}

Table 1: Source Population ${ }^{3}$

\footnotetext{
${ }^{3}$ Sy1 class includes Sy1, 1.1, and 1.2. Sy1.5 class includes strictly Sy1.5. Sy2 class includes Sy1.8, 1.9, and 2. All other sources were classified as others.
} 


\begin{tabular}{cc}
\hline \hline Classification & K-S P-Value \\
Sy1 \& Sy2 & 0.027 \\
Bright Sy1 \& Sy2 & 0.074 \\
Faint Sy1 \& Sy2 & 0.260 \\
\hline \hline
\end{tabular}

Table 2: Kolmogorov-Smirnov P-Values

\begin{tabular}{|c|c|c|c|c|c|}
\hline & Type & Source Count & Fit Method & $\begin{array}{l}\Gamma \\
\end{array}$ & $\sigma$ \\
\hline \multirow{6}{*}{ All Sources } & \multirow{2}{*}{ Sy1 } & \multirow{2}{*}{89} & $W A$ & $1.945 \pm 0.263$ & - \\
\hline & & & $\mathrm{BL}$ & $2.049 \pm 0.036$ & $0.342_{-0.033}^{+0.48}$ \\
\hline & \multirow{2}{*}{ Sy1.5 } & \multirow{2}{*}{16} & WA & $1.939 \pm 0.152$ & \\
\hline & & & BL & $2.070 \pm 0.103$ & $0.404_{-0.062}^{+0.084}$ \\
\hline & \multirow{2}{*}{ Sy2 } & \multirow{2}{*}{68} & WA & $1.851 \pm 0.166$ & \\
\hline & & & $\mathrm{BL}$ & $1.927 \pm 0.033$ & $0.274_{0.022}^{+0.025}$ \\
\hline \multirow{6}{*}{ Bright Sources } & \multirow{2}{*}{ Sy1 } & \multirow{2}{*}{17} & WA & $1.943 \pm 0.169$ & \\
\hline & & & $\mathrm{BL}$ & $1.985 \pm 0.060$ & $0.243_{-0.36}^{+0.048}$ \\
\hline & \multirow{2}{*}{ Sy1.5 } & \multirow[b]{2}{*}{9} & WA & $1.938 \pm 0.168$ & \\
\hline & & & BL & $1.935 \pm 0.042$ & $0.123_{-0.024}^{+0.036}$ \\
\hline & \multirow{2}{*}{ Sy2 } & \multirow{2}{*}{24} & WA & $1.851 \pm 0.166$ & \\
\hline & & & BL & $1.940 \pm 0.048$ & $0.235_{-0.030}^{+0.038}$ \\
\hline \multirow{6}{*}{ Faint Sources } & \multirow{2}{*}{ Sy1 } & \multirow{2}{*}{72} & $\overline{\mathrm{WA}}$ & 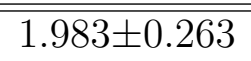 & \\
\hline & & & BL & $2.090 \pm 0.041$ & $0.351_{-0.027}^{+0.031}$ \\
\hline & \multirow{2}{*}{ Sy1.5 } & \multirow{2}{*}{7} & WA & $2.042 \pm 0.152$ & \\
\hline & & & $\mathrm{BL}$ & $2.223_{-0.215}^{+0.219}$ & $0.549_{-0.121}^{+0.201}$ \\
\hline & \multirow{2}{*}{ Sy2 } & \multirow{2}{*}{44} & WA & $1.914 \pm 0.253$ & \\
\hline & & & BL & $1.920 \pm 0.044$ & $0.293_{-0.029}^{+0.034}$ \\
\hline
\end{tabular}

Table 3: Mean Photon Indices of Source Population using Gaussian Fit ${ }^{4}$

\footnotetext{
${ }^{4} \mathrm{BL}$ and WA stand for binned likelihood and weighted average, respectively.
} 


\begin{tabular}{rcccccc}
\hline \hline & & & & & \\
& Type & Source Count & $\Gamma$ & $\mathrm{E}_{C}[\mathrm{keV}]$ & $\mathrm{R}$ & $\chi_{\nu}^{2}$ \\
\hline & & & & & & \\
Sy1 & 89 & $2.143 \pm 0.07$ & - & - & 1.38 \\
Sy 1 & 89 & $2.079 \pm 0.03$ & - & $0.675_{-0.20}^{+0.26}$ & 1.04 \\
& Sy 1 & 89 & $1.522_{-0.07}^{+0.16}$ & $81_{-11}^{+39}$ & - & 1.01 \\
\cline { 2 - 7 } & & & & & & \\
& Sy 2 & 68 & $2.035_{-0.10}^{+0.06}$ & - & - & 1.83 \\
Sy 2 & 68 & $1.956_{-0.04}^{+0.05}$ & - & $1.779_{-1.09}^{+2.07}$ & 0.912 \\
& Sy 2 & 68 & $1.310_{-0.12}^{+0.05}$ & $74_{-12}^{+7}$ & - & 1.27 \\
Sy 2 & 68 & $1.793_{-0.22}^{+0.23}$ & $360_{-46}^{+156}$ & $1.102_{-0.30}^{+0.49}$ & 1.11 \\
\hline
\end{tabular}

Table 4: Fitting Stacked Spectra with PEXRAV Model 